\title{
Support Vector Machine
}

National Cancer Institute

\section{Source}

National Cancer Institute. Support Vector Machine. NCI Thesaurus. Code C78542.

A classification method that will construct a hyperplane between two sets of data such that it maximizes the margin between the hyperplane and the nearest samples. 